\title{
The Application of Traditional Chinese Medicine to Western Psychotherapy: A Case Study
}

\author{
Chunhua Shih', Mingjen Yang², Jungkwang Wen ${ }^{3}$ \\ ${ }^{1}$ Department of Life and Death, Nanhua University, Chiayi County, Taiwan, China \\ ${ }^{2}$ Dr. Yang Ming-Jen's Psychiatric Clinic, Kaohsiung City, Taiwan, China \\ ${ }^{3}$ Wen Xin Psychiatric Clinic, Kaohsiung City, Taiwan, China \\ Email: xianzhenglu2581@yahoo.com.tw,jkw.pc@msa.hinet.net
}

How to cite this paper: Shih, C. H., Yang, M. J., \& Wen, J. K. (2020). The Application of Traditional Chinese Medicine to Western Psychotherapy: A Case Study. Open Journal of Social Sciences, 8, 265-281.

https://doi.org/10.4236/jss.2020.85019

Received: April 29, 2020

Accepted: May 22, 2020

Published: May 25, 2020

\begin{abstract}
Background: Western psychological practices have traditionally treated only the mind, not the body. By contrast, traditional Chinese medicine (TCM) treats both body and mind as a single gestalt. This paper presents a single case study where Western and TCM therapies were integrated into a short-term structured treatment. Study Population: One male client suffering from neurotic depression and anxiety manifesting in both emotional and physiological distress, including insomnia and diarrhea. One therapist participant trained in both Western psychiatric practices and TCM. Method: A course of treatment was devised combining Western psychotherapeutic technique and TCM's diagnostic and therapy practices. Modern methods of focusing psychotherapy, narrative, and mindfulness were used to determine the specific traumatic events causing the patient's issues. Treatment was undergone by "thought imprint therapy" and finally concluded using "psychological acupuncture". Results: The patient reported that his emotional and physical symptoms were greatly alleviated. He was able to sleep without medication and his anxiety towards taking exams was gone. Conclusion: Although a single case study, we are optimistic that this integrative method has great promise for future psychiatric practice.
\end{abstract}

\section{Keywords}

Psychotherapy, Traditional Chinese Medicine (TCM), Low Resistance Thought Imprint Therapy (TIP), Psychological Acupuncture

\section{Introduction}

With modern developments and rapid advancements in technology come sig- 
nificant life changes. Emotional problems arise with increases in pressure from our personal lives and workplaces. Mood swings occur under such conditions, oscillating between emotions such as delight, anger, sorrow, and happiness. We have known for a long time that mental health greatly impacts physical health, as well as our daily lives and work.

Emotions are a type of inner experience, a way for people to react and respond towards objective reality. Emotional reactions are highly complex phenomena and involve multiple neurophysiological systems. In Western medicine, these systems include the nervous, muscular, and endocrine systems. In Traditional Chinese Medicine ("TCM") they are the zàngfŭ, the so-called Five Viscera and Six Bowels (Lai, 2016). However, when treating a client with a mental illness, it is necessary to consider both the physiological and psychological etiologies for their condition and to closely to connect them with the client's social life.

In modern clinical practice, treatments for patients with emotional disorders have traditionally relied on the two pillars of psychotherapy and medication. However, societal changes and restrictions on health insurance have gradually phased out long-term psychotherapy. Patients today often seek to solve their problems with only a few consultations, or even a single session. As a result, current treatments often rely primarily on drug therapy, which focus on improving the client's physiological state and rapidly controlling their symptoms. However, the side effects of the drugs present problems of their own and thus limit the efficacy of such treatment.

The theory of emotion in TCM involves changes between the polar Yin-Yang forces and the so-called Five Elements within the Five Viscera (aka, the "Yin" organs) and the Six Bowels (aka the "Yang" organs) of the zàngfú. This theory presents another way to explore psychopathology, one that is convenient and can supplement Western medical thinking. By integrating both schools of thought into short-term structural psychotherapy, we can treat both body and mind as a single gestalt according to two concepts: "Reducing surplus to relieve deficits" and "No pain, no blockage; and no blockage, no pain." By conducting such treatment under a high level of empathy, the benefits of therapy could be realized while reducing both duration and cost.

The purpose of this case study is to apply the integrated psychotherapies of Chinese and Western medicine to the treatment of patients diagnosed with mild mental disorder in a psychiatric outpatient clinic by using a short-term structured approach to assess the improvement of physical and psychological symptoms and explore the treatment process with in-depth interviews to assess its feasibility.

\section{Diagnosis by Both Traditional Chinese Medicine and Western Psychology}

Differences exist between Traditional Chinese Medicine and Western psychology on diagnosis, etiologies, and the treatment of psychological disorder. However, each also has their own strengths. Therefore, to be able to draw upon each oth- 
er's merits would be of great benefit in a clinical setting (Chung, Lin, Lee, Lee, Lin, Yang, \& Yang, 2007).

Although this article focuses primarily on TCM concepts of psychology, they are placed within the contexts of Western medicine, psychology, and psychiatry to better facilitate understanding by modern readers.

\subsection{The Four Diagnostic Methods of TCM: Visual Inspection, Sound and Smell, Inquiry, and Palpitation}

TCM practitioners collect client information from four different perspectives to form a basis for diagnosis and treatment:

1) Visual Inspection: Western psychologists routinely make observations about the overall appearance, form, look, actions, and reactions of the client. If the client is accompanied by a companion, then observations about the companion and about the interactions between client and companion are also made.

In addition to the above, further assessment of the psychological state of the patient would be also through examinations of the tongue, the ears, and other body parts.

2) Sound and Smell: Listen to the client's voice and observe any smells that may accompany them. Make note of the client's expression, as well as their overall body language. Further valuable information can often be obtained from the client's smell, as well as any statements they make about their sense of taste.

3) Inquiry: Ask questions to explore the ins and outs of the client's problems and to find clues and insights into the client's symptoms and problems. Through inquiry, seek information about the situation and circumstances surround the client's condition, their experiences in seeking assistance, their memories of the past, etc. The information obtained would be used as the basis for diagnosis and to create a treatment plan.

4) Palpation: This refers to diagnosis by touch. The practitioner uses their hand to feel the client's body, assessing the pulse and body surface temperature in each part of the trunk and the extremities. It is possible to obtain information about the client's state of mind and body through changes in pulse and body temperature, which can form the basis for the assessment of the balance of the client's Yin-Yang and Five Elements forces.

\subsection{Combining Perspectives from Both the Chinese and Western Medicine to Determine the Underlying Cause of Illness}

In both TCM and Western medicine, depression is caused by psychological trauma (Chen, 2005). In TCM, such trauma can affect the functions of the emotions that correspond to each of the Five Viscera (the Yin organs) (Lai, 2016). The cause of mental illness is related to the intensity and duration of such emotions, but the more important determinant is the strength of the affected organs. In TCM, the fluency of Qi, is the cause of emotional (mood) disorders. When one's qi (氣, vital energy) is "constrained" or "depressed", it causes a series of changes in one’s blood, "fire element” (huǒ, 火), and "body fluids" (jīnyè, 津液), which 
lead to disturbances in the Creative (Generating) and Destructive (Control) Cycles of the Five Elements, imbalances between Yin and Yang, which manifest as symptoms in the corresponding Five Viscera (Yin organs) and Six Bowels (Yang organs) (Chen, 2005).

In Western medicine, depression, anxiety, obsessive-compulsive disorder, trauma, stress disorder, etc., are all classified differently with different diagnoses. The diagnostic criteria describe the symptoms and course that the illness may take and classify mental illnesses into different categories. Etiologically speaking, different diagnoses reflect disordered states of various neurotransmitters (Chen, 2005). Collectively, this means that brain dysfunction causes mental activities such as cognition, emotion, consciousness, behavior, etc., to manifest as mental illness of varying degrees.

To a certain extent, the TCM model for changes in emotions via Yin-Yang and Five Element interactions corresponds to the changes in brain structure and chemistry under trauma and irritability. The occurrence of such changes (in TCM) are nearly analogous to phenomenon observed in the limbic system and brainstem of neural reactions induced by stress.

In recent years, the term "autonomic dysregulation" (aka "dysautonomia") has been widely used in the field of psychiatric medicine. Broadly speaking, dysautonomia is a condition in which the autonomic nervous system does not function properly. It basically describes the influence caused by the unstable state presented when the amygdala (a component of the limbic system responsible for emotional responses) is overstimulated. This then leads to the dysfunction of the locus coeruleus (a cluster of neurons involved in stress and panic responses and an activator of the sympathetic nervous system) and the vagus (nerves originating in the medulla oblangata which play several regulatory functions) (Chen, Zhang, Liu, \& Huang, 2018).

Hyperstimulation of the sympathetic nervous system can suppress some of the vagus functions, resulting in distraction and cognitive disorders. The face and throat are affected as well, and this can in turn affect interpersonal relationships. A hyperstimulated nervous system can also changes in one's glands (creating hormone and neurotransmitter imbalances) and muscles and internal organs (creating other negative physiological responses).

In TCM, the "Liver" is not just the anatomical organ, but a concept and a dynamic body system based on the anatomical liver. "[It] is in charge of dispersion and dredging; it is mainly responsible for the regulation of emotion, the promotion of digestion and absorption, the maintenance of the circulation of qi, blood, and body fluid, as well as the reproductive function." (Liu, Shu, Tu, Zhang, \& Hong, 2017). In TCM, several emotional disorders and syndromes are connected to the Liver, e.g., insomnia, depression, amentia, irritability, and dizziness.

In addition, Liver stagnation, mental torment, the production of phlegm from the jinyè body fluids, mental disturbances, and the obstruction of the orfices (including tongue, ears, eyes, nose, mouth, genitalia, and anus) are also common etiologies resulting in mental illness (Chen, 2005; Lai, 2016). 
According to TCM, the seven emotions are joy, anger, worry, pensiveness, grief, fear, and fright. These emotions are all manifest in normal, healthy mental activity; however, an excess of any of the emotions will affect normal mental activity and lead to mental illness. Such illnesses are classified into five general categories that correspond to the concepts of Creation and Destruction in Yin-Yang and Five-Element theory (also known as "Generation" and "Control"). The "Five Viscera" are the "Five Zang Organs" (wǔzàng, 五藏), which are the Liver, Heart, Spleen, Lung, and Kidney "Organs" (again, concepts and dynamic body systems based on the anatomical organs). These Organs respectively correspond to the Wood, Fire, Earth, Metal, and Water Elements, and each "hide" (contain) both “essence” (jīng, 精) and "spirit” (shén, 神). "Hiding” in the Liver is Hún (魂, "soul”); in the Heart, Shēn (神, here meaning "mind”); in the Spleen, Yì (意, “intent”); in the Lungs, Pò (魄, “vitality”); and in the Kidneys, Zhì (志, “willpower"). These "Five Spirits" (wǔshēn, 五神) resonate with the Five Viscera, the wǔzàng, and as such, excessive "emotions" harm each of these Viscera: anger harms the Liver, joy harms the Heart, pensiveness harms the Spleen, grief harms the Lungs, and fear harms the Kidneys (Chen, 2005; Lai, 2016).

When a mood swing causes changes in a person's Yin-Yang and Five Elements, what emerges are various physiological phenomena controlled by the autonomic nervous system. Ancient Chinese medical practitioners and theoreticians observed these changes and phenomena and used Yin-Yang and Five-Element theory to create categories for diagnostic classification. Treatments are based on the practices of “syndrome/pattern differentiation" (biànzhèng, 辨證) and “qi patterns” (biànqì, 辨氣). Therefore, by recognizing how neuroanatomy affects physiological change and function, and by comparing Chinese and Western medical theories about the relationship between the body, the mind, and emotions, we may find that "sweet spot" where we can successfully combine both Eastern and Western medical thought.

\section{Psychotherapy Techniques that Integrate Both Chinese and Western Medicine}

\subsection{Principles of an Integrated Psychotherapy}

1) "No pain, no blockage; no blockage, no pain." Clear obstructed channels to relieve stagnation and remove traumatic experiences.

The root cause of "depressed" qi can be understood as a physiological change in response to various acute or chronic threats to one's life and safety.

In Buddhism, the Eight Duhkha ("Sufferings") arise from birth, old age, sickness, death, meeting with those whom one hates, unfulfilled desire, and the Five Skandha ("Aggregates", the material and mental forms that lead to craving and clinging) (Wang, 2010). The Eight Sufferings are interpreted as a variety of situations in everyone's lives in which each person must face the feeling of insecurity. They are derived from humanity's fear of said insecurity and death and are reflected in the parenting in the parenting process. In other words, how parents 
interact and raise their children is related to how their offspring will react to insecurity in the future.

In Western psychology, there is the potential for the parenting process to cause a wide range of traumas, such as attachment, narcissism, object relations, impermanence, lack of self-control, separation anxiety, the Oedipus complex, etc. If a child's psychological needs cannot be fulfilled, most will transmute into thanatophobia, the fear of death, and such drives and fears will dominate that person's life. Through the full processes of seeking one's fortune while avoiding evil and of ridding oneself suffering while finding happiness, one gradually forms coping mechanisms while experiencing the suffering of life (Oyang \& Wu, 1998).

In TCM, long-term illness may impact the luòmài (絡脈, the "collateral vessels"). The luòmài interact with the jīngluò (經絡, the "channel network"); together, they are collectively referred to as the "meridians" and acupuncture points, and they are where information on "long-term stagnation" is stored and may be found. By understanding the interaction between mind and spirit, and by using syndrome differentiation through Yin-Yang and Five-Element theory, we are able to see the cause-and-effect relationship between memories of past trauma and symptoms of contemporary disease. This may then be used to determine the most appropriate treatment with the best curative effect ( $\mathrm{Li}, \mathrm{Yang}$, \& Wang, 2004).

In clinical practice, one can see-through the four diagnostic methods of visual inspection, sound and smell, inquiry, and palpation-that "body memory" and other such information are stored in the meridians and acupuncture points. In particular, palpation and corresponding inquiry can reveal recessive symptom-related memories concealed within the body. In other words, there are both theoretical and practical bases for combining the physical therapy of Chinese medicine and Western speech therapy to relieve traumatic memories (Raabe, 2010).

2) The Balance of Yin and Yang- "Reduce surplus and supplement deficiency"

Balance (平, píng) and Moderation (中庸, zhōngyōng, also translated as "equilibrium" or "mean") are principles used in TCM psychotherapy techniques (Fu, Wang, Tang, Liao, \& Wang, 2012). The biggest consideration of any treatment in TCM is to keep or restore the balance of Yin and Yang by "reducing surplus" or "supplementing deficiency", often accomplishing the latter by accomplishing the former. After the four diagnoses (which are outlined above), it is necessary to determine how to relieve excess emotions, how to provide empathy and support, and how to apply the appropriate treatment. This is performed using biànzhèng syndrome differentiation and identifying biànqì qi patterns as the basis for treatment.

3) Empathy is the foundation of treatment

Empathy is at the foundation of all therapy. It is the most important factor in all psychological techniques, and only with empathy can treatment be carried out smoothly (Wang, Bai, \& Zhang, 2007). 
Generally, reflect is necessary after inquiring about the client's inner conflicts, emotional state, and expectations from therapy. It is aimed at the client's cognition, emotions, and behaviors. Focusing techniques begin by the therapist gauging the client's physical state, the therapist reflecting the client's verbalized thoughts and feelings back to the client, and the client using those reflections to as a cross-reference to achieve a Gendlin's "felt sense", a realization that moves the therapy forward. Such concepts illustrate modern psychotherapy's willingness and need to pay attention to the body, not just the mind ( $\mathrm{Wu}, \mathrm{Xu}, \&$ Wang, 2016).

4) "The present moment" is the field of healing

The ultimate key to treatment is that both the client and the therapist can both be in the present moment in order to form an "us". The concept of "us" is the foundation of all relationships. The concept of "us" is emphasized in "relationship", a connection through which a sense of recognition and belonging can be achieved. If patients and doctors can connect with each other and create a good medical relationship, the treatment is bound to yield greater benefits (Huang, 2018). That is, the flow of mindfulness between the two connected persons and the effort to understand each other produce the initial healing effects. Psychotherapy work is also built on this foundation, so it is important to let it flow naturally! Therapy can only be effective if there is a natural connection, a flow, through which empathy, resonance, and attunement can lead to personal transformation and change and generate forward moment for healing.

The most important thing in psychotherapy is this relationship, as everything stems from it. The "us" comes from the relationship created by the interaction between therapist and client. Good interactions inform the client's inner presentation and maintains a good connection between therapist and client. "Empathy" is the focus of therapy, which fundamentally requires a good relationship (Hsing \& Chen, 2018). This connection cannot definitely not superficial; it must a real link. Therefore, the key to healing is to allow the client to be aware, understand and experience the changes in his heart, in the present, and then to experience them.

\subsection{Techniques of Psychotherapy in This Study}

1) Mindfulness

The most important thing about mindfulness is that it allows one to break out of the automatic (or "triggered") pattern of cognition and achieve a completely different awareness in the present. Over time, mindfulness-based therapy has shown considerable ameliorative effects on neuroses, anxiety disorders, schizophrenia, panic disorders, depression, obsessive-compulsive disorder, post-traumatic stress disorder, and chronic pain (Tang, 2018).

2) "Focusing"

Continuing the legacy of humanism, Eugene T. Gendlin took psychotherapy to a new level by establishing the technique of "focusing psychotherapy". Focus- 
ing psychotherapy not only contains the spirit of humanism but also a similarity of Buddhism (Wu, Xu, \& Wang, 2016).

In focusing, these repressed contents of unconsciousness, represented by any forms of symbol, metaphor, and physical symptom, are waiting for further exploration, operation, and then become life experience, which are known as "handles". Listening plays a very important part in focusing. The listener reflects the client's thoughts, emotion and behaviors back, and through this, the "mystery of Vipassana" (insight through self-observation) can reveal the root of the patients' distress and symptoms. At this moment, the symptoms become a "living" subject to the client, not just an object that "is". By changing the relationship between the client and their subject, a deeper experience is produced. It then becomes easier to gradually and instantly reveal the Bion's "beta state" of the consciousness, and thus present them through a various of physiological changes, symbolic images, or metaphors (Lu, 2010). Focusing is a slow process that uses the subjective experience of the one who is "focusing" as the main process of work, and the listener only has to follow the experience without intervention, allowing the repressed consciousness to flow smoothly (Wu, Xu, \& Wang, 2016).

3) Narrative techniques

Experience is a constructed model occurring during the process of interaction between the individual's cognitive schema and the impact of the environment on her/himself; Narrative is to explore the typical story script of the social context in which the individual is located, and the potential new story script that may be hidden allows one to see new story lines beyond the typical story script in a new frame of reference. The new framework means new experiences and meanings, thereafter a different awareness of life will also be felt (He, 2006).

4) Low Resistance Thought Imprint Therapy (TIP)

Thought imprint therapy is a TCM psychotherapy technique developed by Wang Weidong (Liu \& Wang, 2019). In simple terms, the therapist uses special methods to generate different states of consciousness, allowing the therapist to establish a connection to the client's unconsciousness and then to directly communicate and coordinate with it to change the client's unconscious.

A "low resistance" state can be interpreted as a hypnotic state, one which may be aided by the use of verbal cues or hypnotic techniques. It is an intermediate state between wakefulness and sleep; the goal of thought imprint therapy is to establish with a kind and positive transference relationship under the low resistance state. Therapy is then conducted under these conditions, where the conscious mind is stripped away and the therapist can work with different cognitive levels to achieve the goals of treatment. It is consistent with the nature of psychological counseling while also being different.

5) Psychological Acupuncture (Zhang, 2019)

Psychological acupuncture is a fusion of Western psychology and TCM by Zhang Shouchun. It was formulated on the bases of the "13 Ghost Acupuncture Points" (used since the Tang dynasty to treat psychological ailments); 81 "psychological points" selected from the standard 361 along the 14 meridians; and a 
combination of modern sciences, such as psychology, bio-holographic theory, psychoneuroimmunology, and neural plasticity. This fresh and convenient approach to psychotherapy is currently practiced only in China.

\section{Materials and Methods}

\subsection{Selection of Participants}

Client Participants:

By purposive sampling, psychiatric outpatients met the ICD-10 criteria for neurotic depression and/or anxiety and were currently using psychiatric drugs who without receiving any counseling, were recruited for his study.

Therapist Participants:

Researchers were selected who fulfilled both of the following criteria:

1) Having trained in TCM and TCM psychotherapy, and

2) Being a psychiatrist currently engaged in consultations or treatment of clients.

\subsection{Research Methods and Procedures}

The major method used in this study was a combined TCM/Western short-term structured psychotherapy and was based on the following techniques: the four TCM diagnoses of visual inspection, sound and smell, inquiry, and palpation; Wang's thought imprinted therapy; Zhang's psychological acupuncture; and narrative, mindfulness, and focusing techniques. In-depth interviews were conducted after treatment to assess its efficacy.

\subsubsection{The Combined Chinese/Western Short-Term Structured Psychotherapy Was Divided into Three Phases and Six Individual Steps}

1) Initial phase: Conduct a back-and-forth inquiry to assess the client's emotions and establish a trusting and safe doctor-patient relationship

a) Confirm the main issue (證, zhèng, "pattern" or "syndrome") through a description of the symptoms.

b) Build a safe and trusting doctor-patient relationship.

c) Establish a preliminary diagnosis and treatment plant, using focusing techniques.

2) Treatment phase: Introduction of thought imprint therapy

a) Conduct Wang's thought imprint therapy after establishing an empathetic relationship that emphasizes "us"-ness, security, and trust.

3) Consolidation Phase: Establishing new cognitive schema

a) Complete the treatment using Zhang's psychological acupuncture to help defuse past traumas and build new neural circuitry.

The entire course of treatment was conducted over six sessions, and the content of each session could be adjusted on a case-by-case basis.

\subsubsection{Reviewing the Process of Treatment}

Both client and therapist participants were interviewed separately after completion of the course of treatment. Prior to the interview, the researcher was to ex- 
plain to both parties that the information obtained from the interview would be kept confidential and that the interview would be conducted separately from therapy. If either the client or the therapist expressed negative feelings towards the other party, the researcher was to remain neutral and listen without any giving any response, questioning, or intervening.

The separate treatment review interviews were conducted within three days of the completion of the full course of treatment ( 3 phases, 6 steps) as follows:

Please describe what you thought and felt about the treatment as a whole before you entered this session.

Please describe your/the client's physical and emotional condition before you entered this session, depending on whether you were a client participant or a therapist participant.

1) Please describe any changes you experienced in your therapeutic relationship since entering this treatment, and what you were thinking and feeling at the time of said changes.

2) Please describe the moments that made an impression on you throughout the entire course of treatment, from beginning to end.

3) Please describe how you have/the client has changed physically and emotionally since you completed this course of treatment.

4) Please describe the moments that made an impression on you throughout the entire course of treatment, from beginning to end.

5) Please describe how you have/the client has changed physically and emotionally since you completed this course of treatment.

\section{Results}

\subsection{The Client's Background}

Male, 33 years old at the time of treatment, who is unmarried with a medical degree, currently living alone. He has an older sister.

The client's father died of myocardial infarction in 2011. In 2012, his mother fell to her death after an overdose of hypnotics. The client is the youngest child in the family. His childhood and adolescence were mainly spent with his grandparents. At a young age, he witted domestic violence of his father toward mother. The father's immediate family was not well-off, but the mother's was quiet affluent. The parents' relationship began to noticeably degrade when the client was in the seventh grade. His parents often argued over money and became violent towards each other. In addition, the father changed jobs frequently because of conflicts with his bosses, which caused financial problems and considerable frustration.

The client's parents divorced when he was a university freshman (1995), and he decided to retake his entrance exams and apply for the medical school. Life at that time was described as "difficult." Since childhood, the client was closer to his mother than his father. After graduating from medical school, preparations for the national licensing exam did not go well. At present, in addition to preparing for the national exam, the client makes a living by tutoring. 
The client described his sister as "grumpy" and "hard to get along with", and he often has conflicts with her. Her processional certification exams did not go well, either. The client spent the Chinese New Year holiday with his sister, but they got into several conflicts during this period.

In the past, the client had a girlfriend with whom he dated for four and a half years. Her mother has a strong personality, wanted to intervene in everything between the couple. He became intolerable and finally the affair broke when he was an intern.

The client had sleeping problems in high school. In the years leading to medical school, he found himself easily getting nervous, and his physical condition deteriorated. The client often became ill and caught colds repeatedly. During that time, his parents divorced, which cause a turbulent family situation. The client didn't seek medical help until the accidental death of his mother exacerbated his symptoms to the point that he was only sleeping once every $2-3$ days.

\subsection{The Three-Phase/Six-Session Treatment}

The client's main issues were an extreme fear of facing his exams, which manifested as severe physical and mental weakness, severe insomnia, and diarrhea. During focusing therapy, it was determined that the main traumas were his past exam experiences and learning about his mother's death. After some discussion, it was decided that goal of treatment would be to alleviate his exam anxiety, and after assessment thought imprint therapy and psychological acupuncture therapies would comprise his course of treatment. After the completion of the three phases and six steps, interviews were conducted to assess the client's and the therapist's experience with the applied treatment. The client's on-going prescription for high-dosage sleep aids was continued through the treatment course.

At the end of the third session (preliminary diagnosis using focusing techniques and establishing a treatment plan), the client tearfully exclaimed, "Wow! I've never felt so relaxed in my life!" In subsequent sessions, the client smiled more and participated more actively in talks with the therapist, indicated great improvements in sleep and other psyschosomatic conditions, and most importantly, the courage to study and concentrate on passing his exams. After the fifth session, the client said that he could now sleep without sleep pills.

\subsection{Changes through the Process of Therapy}

\section{Client's perspective}

Changes in the client's perspective throughout therapy were summarized as follows:
a) Changes in affectionate relationships
b) Changes in cognitive behaviors
c) Changes in emotional symptoms

1.1. Changes in affectionate relationships

1) From strange/unfamiliar to familiar:

The main points from the interview: 
"I came back to Dr. X again, although I felt scared and anxious about returning for the first time."

"I had great expectations for Dr. X this time, but I was certain if he could actually help me.”

"Upon first seeing of Dr. X, I felt a feeling of familiarity."

2) Being respected and understood:

The main points:

"I feel respected and accepted in every session. I was able to freely share all my feelings and thoughts. No matter what I said, I felt understood by Dr. X from even just the look in his eyes. In situations where I was not able to express with my own words, Dr. X would help me say it, making me feel that he understood all of my sufferings. That's so different from my past experiences talking with someone else."

"Dr. X detected the extreme fear that had been in my heart that I myself hadn't recognized before."

3) Security and trust:

The main content:

"I felt at ease with familiarity, I finally found help."

"In terms of uttering things that I wasn't not capable of dealing with, I felt comfortable discussing them here."

"Dr. X was very professional; I believed that he would find a way to help me."

\subsection{Changes in cognitive behavior}

1) Cognitive changes:

The main points:

"I will never be successful in that I'm lacking competitiveness."

"For me, problems are not solvable according to my own will."

"I have always failed. I have always believed that I'm just not capable."

"I used to look for my shortcomings instead of my merits; I was afraid of failing, and I had no confidence. But now, I know that I'm have value.”

"It cannot get any worse, so just do it, and there will be a chance of success."

"My fear has vanished completely; I'm no longer haunted by negative thoughts."

2) Behavioral changes:

The main points:

"Before therapy, I didn't want to do anything. I just laid in bed in my messy home."

"After therapy, I started to clean the house and looked for a brighter place to move into." 
"I started to exercise."

"I'm at rock bottom. However, if I give things a try, I can only give myself a chance to succeed."

"Now I feel completely at ease, the experience I have never had before."

\subsection{Changes in emotional symptoms}

The main content:

"I was waking up in a state of panic."

"It was difficult for me to fall asleep at night, even with the help of medicine."

"I had severe headaches during the day, which was very irritating."

"I've stopped taking sleeping pills at night for few days, and now I can fall asleep naturally. Also, I have reduced my dosage."

"Significant drops in the intensity of my tension and anxiety, to levels that are bearable."

"With the help of therapy, I had made great improvements in my emotional state, especially the part about being scared. Additionally, my thoughts have changed as well."

"I am free of any medications."

\section{The therapist's perspective}

Changes in the therapist's perspective throughout the therapy can be summarized as follows:

1) Changes in affectionate relationships

2) Changes in cognitive behavior

3) Changes in emotional symptoms

\subsection{Changes in affectionate relationships}

1) From strange/unfamiliar to familiar:

"The first time I saw him, he was very nervous, and he could not look me straight in the eye."

"He was reluctant to say what he desired to express, as if he was afraid of revealing the reason why he was coming back for another visit. He was probably concerned about the possibility of my rejection."

"After my consent to help, his nervousness was replaced by smiles; I see the "he" in my memory coming back."

2) Being respected and understood:

"He was vulnerable and afraid of being rejected, so the first step was to build up the relationship between us was to make him feel respected and understood."

"Let him know that he is understood and has my empathy, to a profounder extent than he had ever experienced before."

"I could tell that my client had a stronger connection with me knowing how I empathized with his suffering." 
3) Security and trust:

"He was reluctant to say what he desired to express, as if he was afraid of revealing the reason why he was coming back for another visit. He was probably concerned about the possibility of my rejection."

"I promised to help him and suggested that medication is not a long-term solution. I asked if he would consider giving the combined Chinese/Western short-term psychotherapy a try. He agreed without hesitating."

"Our relationship became closer when he told me that I discovered how his great fear of being defeated had deepened in his heart when he himself didn't even realize it."

\subsection{Changes in cognitive behavior}

1) Cognitive conversion:

"I used to think that psychiatry and psychology are the only ways for solving psychological problems. Yet, after looking into it, I found that, in traditional Chinese medicine (TCM), psychological issues were not set apart from others that comes from the body. There is no difference between treating either physical or psychological illness in Chinese remedy.”

"My former knowledge of TCM consisted of either medicine too bitter to be swallowed or terrifying acupuncture. I did not expect that psychological problems could be solved by TCM."

"Psychotherapy with TCM is effective and efficient, and free of side effects." "Modern Western medicine is often drug-dominated, whereas psychological therapy is time-consuming. So if we integrate TCM with Western practice, we could actually make up for the disadvantages that came from only using modern Western medical practices."

"TCM and modern Western medicine can be perfectly integrated and complementary in clinical psychotherapy."

2) Behavior conversion:

"I now prefer to treat patients with a combined treatment of TCM and modern."

"Effective treatment without drugs application in today's drug-dominated treatment orientation has become more favorable for clinical needs."

"It will be a main option for me when considering how to treat patients with psychiatric disorders."

"Compared with traditional Western psychiatric medicine, which only uses psychology as a method of intervention, Chinese medicine psychotherapy can deal with people's emotional disorders from a broader perspective. Therefore, it is recommended that professional psychologists should pay attention to the TCM and the applications of clinical practice within psychological framework."

\subsection{Changes in emotional symptoms}

"When we came to the end of the treatment course, I was very moved by 
the tears welling up in the client's eyes."

"My confidence continues to grow, and I'm more capable to face new challenges because of the satisfactory outcome of the treatment."

"Owing to the limitations of traditional psychiatry, there's always room for regret when we consider medical services. The over-emphasis of pharmacotherapy has resulted in a struggle between efficacy and side effects. However, incorporating TCM with psychiatric practices, the outcome of this drug-free treat seemed to a significant improvement. It's very exciting for me to have this result."

"With the help of this case and treatment, I am optimistic about the future of psychiatric therapy.

\section{Discussion and Limitations}

This study is about the integration of TCM and Western psychotherapy for short-term treatment. Building up a relationship between the therapist and the client at the very beginning place is critical to the entire treatment. Empathy-by which we mean understanding the unique experiences of others and responding accordingly-is also critical, as it is the vehicle by which the therapist can help the client transition from despair to hope through the power of experience.

In addition, establishing the sense of "us" in a therapeutic relationship is also a fundamental determinant of therapeutic effectiveness. The relationship itself carries an element of healing. However, "going with the flow" is simpler said than done-for example, calm waters often hide deadly whirlpools. Therefore, it is essential in therapy to have the skills in order to maintain the relationship between "you" and "me", and the key is for both sides to be able to form "us" in the "present" during consultation.

Western medicine usually operates under the paradigm of mind-body dualism and as a result, usually explores psychological issues in isolation from the body. However, TCM embraces the unity between form and spirit and the unity of nature and man and therefore operates under the paradigm that there must be interactions between heaven, earth and man. From this, the foundation of physical and mental health in TCM is balancing of the forces of the Five Elements and of Yin and Yang. 2000 years ago, the foundational text of Chinese medicine, the Huángdì Nèijīng ("The Classic of the Yellow Emperor") (Lai, 2016), proposed theory and practice of psychological prevention, psychological diagnosis, and psychotherapy. Chinese medicine has always recognized the importance of psychological factors in treatment, and the body is often used as a guide for diagnosis and treatment.

Chinese and Western medical practitioners have different views on the diagnosis and etiology of mental illness. Furthermore, while there is few psychiatric diagnosis in TCM, TCM contains a considerable body of knowledge of mental illnesses and experience in effective treatments. Could this then mean that different names and treatment modalities can be equally effective in dealing with a partic- 
ular phenomenon? In other words, if the same phenomenon can be recognized under different concepts, can different treatment modalities be administered, and can both be efficacious? Is there an implicit difference between Western and Chinese approaches to mental health, or is it just a question of epistemology?

The most appropriate treatment must be used for every situation. Sometimes, a single technique can be used; at other times, techniques from multiple disciplines can be integrated. Thought imprint psychotherapy, or "TIP", was one technique used in our combined TCM/Western short-term structured psychotherapy study. TIP is an evidence-based treatment that is generally used as a stand-alone technique in modern Chinese psychotherapy, but we blended the concept of "low resistance" (a variant of hypnotherapy) TIP with other schools of treatment.

Therefore, the "field" of work cannot be entirely at the level of consciousness. It is not only possible to imprint new thought with technique TIP. Also in the pre-consciousness, for symbols, metaphors, bodies, dreams and other exhaled subconscious themes, with the integration with other genres of technique the repressed past trauma is possible to be awarded. Finally, in the way of psychological acupuncture to resolve the schema past trauma, and then establishing a new neural circuit, as a consolidation of treatment.

This is only preliminary study presenting but a single case. Although there are insufficient grounds to assert a theory, the results and what was discovered in the process give us confidence to pursue future developments. Psychiatry has always been the target of criticism, and in modern times, that criticism has asserted that mainstream psychiatric practice is reliant on drugs, regardless of harmful side effects.

The integration of TCM and Western psychotherapy appears to be a convenient one and has the potential to be suitable for short-term treatment. Integrated approaches such as ours should be considered for future clinical practices.

\section{Conflicts of Interest}

The authors declare no conflicts of interest regarding the publication of this paper.

\section{References}

Chen, Y. Y. (2005). A Brief Discussion of the Treatment of Depression with Chinese Medicine. Taiwan Journal of Clinical Chinese Medicine, 11, 202-208.

Chen, Z., Zhang, B., Liu, X., \& Huang, S. (2018). Research Progress on the Pathogenesis of Anxiety. Tianjin Journal of Traditional Chinese Medicine, 35, 316-320.

Chung, H. J., Lin, S. C., Lee, H. J., Lee, T. F., Lin, Y. K., Yang, C. C., \& Yang, S. H. (2007). The Study for Nursing Record Standard of Inpatient Care in Traditional Chinese Medicine. Journal of Chinese Medicine, 18, 129-146.

Fu, Y. F., Wang, J. H., Tang, J., Liao, J. Y., \& Wang, Y. S. (2012). Theory and Method Research of Psychotherapy through Chinese Medicine. Journal of Dali University, 11, 68-70.

He, X. S. (2006). Narrative Therapy: A New Paradigm for Social Work Practice. Journal of East China University of Science and Technology (Social Science Edition), 21, 7-11. 
Hsing, C. P., \& Chen, H. J. (2018). The Importance of the Enhancement of the Involuntary Client's Motivation in the Initial Interview. Guidance Quarterly, 54, 57-66.

Huang, L. L. (2018). From Sympathy to Empathy-Is It Possible to Have a Good Physician-Patient Communication without "Fusion of Horizons"? Monthly Review of Philosophy and Culture, 45, 163-179.

Lai, Y. C. (2016). The Emotion Theory in Medical Classic of Huang Di Nei Jing. M.D. Thesis, Chiyi County: Nanhua Unviersity.

Li S., Yang B., \& Wang, Y. Y. (2004). Invasion of Collateral Vessels by Primary Infection: Its Differentiation and Treatment. Journal of Beijing University of TCM, 27, 7-10.

Liu, X. W., \& Wang, W. D. (2019). A Brief Description of Professor Wang Weidong Clinical Experience in Diagnosis and Treating of Insomnia. World Journal of Sleep Medicine, 5, 603-605.

Liu, Z. W., Shu, J., Tu, J. Y., Zhang, C. H., \& Hong, J. (2017). Liver in the Chinese and Western Medicine. Integrative Medicine International, 4, 39-45. https://doi.org/10.1159/000466694

Lu, Y. J. (2010). Bion's Theory of Containment Function and His Comprehension of Psychosis. Journal of Nanjing Normal University (Social Science), 3, 99-103.

Oyang, Y., \& Wu, L. C. (1998). The Intergenerational Transmission Model of Child-Rearing Style and Attachment for Adolescence and Their Mothers in Taiwan. Bulletin of Educational Psychology, 30, 33-58.

Raabe, P. B. (2010). Healing Words: Philosophy in the Treatment of Mental Illness. Monthly Review of Philosophy and Culture, 37, 21-34.

Tang, H. S. (2018). Mindfulness Base Cognitive Therapy Applied to Obsessive Compulsive Disorder. Chinese Group Psychotherapy, 24, 15-21.

Wang, Y. G. (2010). On the Generation and Elimination of Suffering-A Comparison between the Pessimistic Philosophy of Life of Schopenhauer and the Oriental Buddhism. The Silk Road, 2010, 102-103.

Wang, Y., Bai, X. P., \& Zhang, S. P. (2007). Unscramble Empathy Technique in Psychological Counseling. Journal of Chongqing Jiaotong University (Social Sciences Edition), 7, 85-88.

Wu, Y. X., Xu, J., \& Wang, L. J. (2016). Overview on New Progress in the Humanistic Therapy: Focusing-Oriented Therapy. Science of Social Psychology, 4, 93-99.

Zhang, S. C. (2019). Exploration and Practice of Psychological Acupuncture. The $6^{\text {th }}$ International Congress of China Medicine Psychology, Beijing, China, World Federation of Chinese Medicine Societies. 